\title{
Implementation of a Physician-Based Stroke Unit in a Remote Hospital of North-East India-Tezpur Model
}

\author{
Lydia John ${ }^{1}$ Akanksha William² Dimple Dawar ${ }^{2}$ Himani Khatter ${ }^{2}$ Pratibha Singh ${ }^{1}$ \\ Anjana Andrias ${ }^{1}$ Christina Mochahari ${ }^{1}$ Peter Langhorne ${ }^{3}$ Jeyaraj Pandian² \\ ${ }^{1}$ Department of Medicine, Baptist Christian Hospital, Tezpur, \\ Assam, India \\ 2Department of Neurology, Christian Medical College, Ludhiana, \\ Punjab, India

\begin{abstract}
Address for correspondence Jeyaraj Durai Pandian, MD, DM, FRACP, FRCP, FESO, FWSO, FNAMS, Department of Neurology, India (e-mail: jeyarajpandian@hotmail.com).
\end{abstract} \\ Christian Medical College and Hospital, Ludhiana, Punjab 141008,
}

3 Institute of Cardiovascular and Medical Sciences, Royal Infirmary Hospital, Glasgow, United Kingdom

J Neurosci Rural Pract 2021;12:356-361.

\begin{abstract}
Keywords

- stroke unit

- low- and

middle-income

countries

- remote area

- care pathway

- multidisciplinary team

Objective The study aims to determine the effects of implementing stroke unit (SU) care in a remote hospital in North-East India.

Materials and Methods This before-and-after implementation study was performed at the Baptist Christian Mission Hospital, Tezpur, Assam between January 2015 and December 2017. Before the implementation of stroke unit care (pre-SU), we collected information on usual stroke care and 1-month outcome of 125 consecutive stroke admissions. Staff was then trained in the delivery of SU care for 1 month, and the same information was collected in a second (post-SU) cohort of 125 patients.

Statistical Analysis Chi-square and Mann-Whitney $U$ test were used to compare group differences. The loss to follow-up was imputed by using multiple imputations using the Markov Chain Monto Carlo method. The sensitivity analysis was also performed by using propensity score matching of the groups for baseline stroke severity (National Institute of Health Stroke Scale) using the nearest neighbor approach to control for confounding, and missing values were imputed by using multiple imputations. The adjusted odds ratio was calculated in univariate and multivariate regression analysis after adjusting for baseline variables. All the analysis was done by using SPSS, version 21.0., IBM Corp and R version 4.0.0., Armonk, New York, United States.

Results The pre-SU and post-SU groups were age and gender matched. The post-SU group showed higher rates of swallow assessment (36.8 vs. $0 \%, p<0.001)$, mobility assessment, and re-education ( 100 vs. $91.5 \%, p=0.037$ ). The post-SU group also showed reduced complications ( 28 vs. $45 \%, p=0.006$ ) and a shorter length of hospital stay $(4 \pm 2.16$ vs. $5 \pm 2.68$ days, $p=0.026)$. The functional outcome (modified ranking scale) at 1-month showed no difference between the groups, good outcome in post-SU (39.6\%) versus pre-SU (35.7\%), $p=0.552$.

Conclusion The implementation of this physician-based SU care model in a remote hospital in India shows improvements in quality measures, complications, and possibly patient outcomes.
\end{abstract}

published online

February 22, 2021
DOI https://doi.org/

10.1055/s-0041-1723099 ISSN 0976-3147. (c) 2021. Association for Helping Neurosurgical Sick People. This is an open access article published by Thieme under the terms of the Creative Commons Attribution-NonDerivative-NonCommercial-License, permitting copying and reproduction so long as the original work is given appropriate credit. Contents may not be used for commercial purposes, or adapted, remixed, transformed or built upon. (https://creativecommons.org/licenses/by-nc-nd/4.0/).

Thieme Medical and Scientific Publishers Pvt. Ltd. A-12, 2nd Floor, Sector 2, Noida-201301 UP, India 


\section{Introduction}

In the year 2016, globally there were 80.1 million prevalent cases. The incidence of stroke in India ranges from 121 to 150 per $100,000 .{ }^{1}$ There is a high burden of stroke in the north-eastern states of India. ${ }^{2}$ Among the various evidence-based stroke treatments, stroke unit (SU) care has been estimated to have the greatest population impact. ${ }^{3}$ However, establishing SUs in low and middle-income countries (LMICs) is challenging because of limited resources, lack of infrastructure, shortage of neurologists and rehabilitation staff, and lack of government funding. ${ }^{4}$ Various strategies are being studied and explored in LMICs that are culturally acceptable and cost-effective. ${ }^{5}$

Despite these challenges, there has been a rise in the number of SUs in LMICs. ${ }^{6}$ However, further expansion would be greatly aided by the development of a physician-based model of SU that is a sustainable and viable option. This model of care has been tested in SU trials in high-income countries $^{3}$ but is relatively untested in LMIC settings, and it is unclear whether they will improve stroke outcomes. This research was designed to evaluate the effects of implementing a physician-based SU model in a remote hospital in North-East India.

\section{Methods}

This study was a before and after cohort study performed in the Baptist Christian Hospital (BCH) in Tezpur, Assam. The data collection for the pre-SU group was done from January 2015 to March 2016 and for the post-SU group from October 2016 to December 2017. The SU manual was developed, and the staff in the SU was trained during the intervening period. The inclusion criteria for the study followed all patients admitted with ischemic, hemorrhagic strokes, transient ischemic attack, and spontaneous subarachnoid hemorrhage over 18 years of age. Patients with a traumatic brain hemorrhage and brain tumor were excluded from the study. The approval for the study was obtained from the institutional review board of Emmanuel Hospital Association, New Delhi. The informed written consent was taken from all the participants of the study.

Tezpur is situated in the eastern state of Assam in India (-Fig. 1).

According to the 2011 census, the total population in this region is 102,505 . There are 13 hospitals in Tezpur including private and public, and no SUs in these centers. BCH is a 200-bed secondary care hospital with National Accreditation Board for Hospitals and Healthcare Providers accreditation. There is one physician (Department of Internal medicine), besides 27 doctors from different departments (obstetrics and gynecology, ophthalmology, orthopedics, pediatrics, radiology, anesthesia, surgery, and dental), 126 nurses, two physiotherapists, and an occupational therapist in this hospital. There are no neurologists in this region. The hospital has a CT Scanner and facilities to do carotid and vertebral Doppler studies were unavailable during the study period.

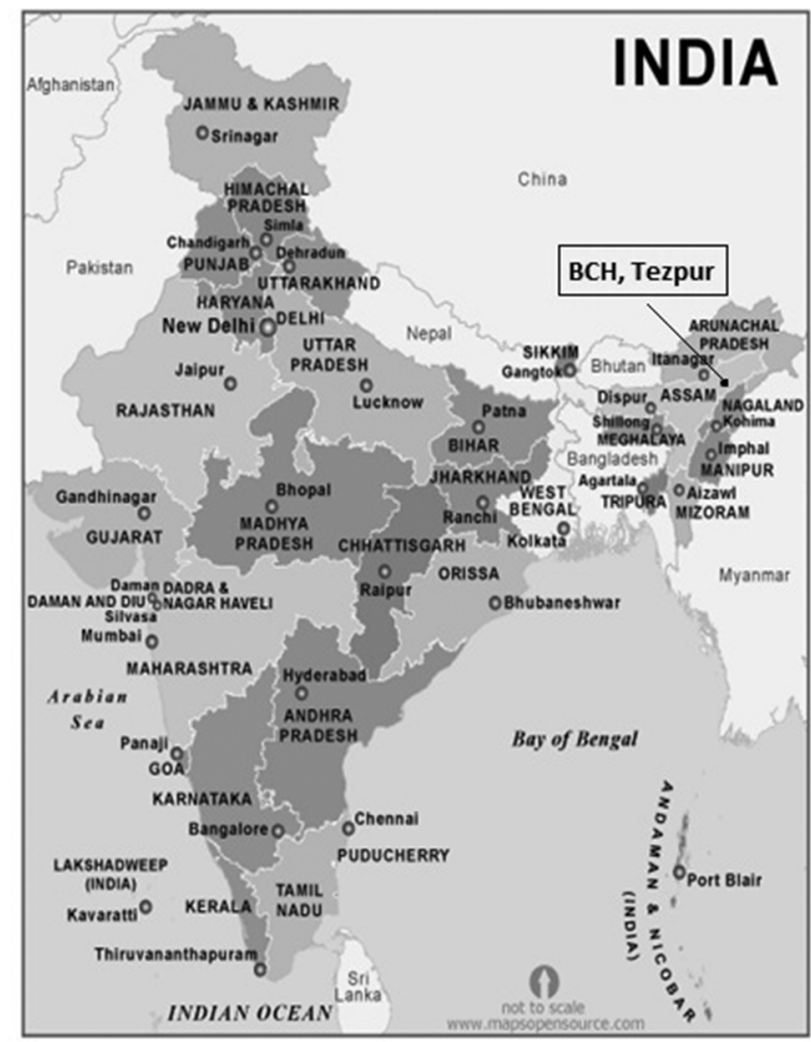

Fig. 1 Shows the Tezpur location on the map of India.

\section{Pre-Stroke Unit Phase}

Before implementing the SU intervention, we collected information on a cohort of 125 consecutive stroke admissions. All the demographic information was collected from patient charts by the research staff in $\mathrm{BCH}$.

All stroke patients were admitted and treated in a medicine ward. The nurses allotted in these wards were not specially trained to care for stroke patients. Antiplatelet, anticoagulant medication prescription, deep vein thrombosis prophylaxis, and swallow test were not routinely performed on all patients. Variables like the National Institute of Health Stroke Scale (NIHSS), medical complications, multidisciplinary team (MDT) approach, and length of hospital stay were collected. Patients were also followed up at 1 month and the outcome was documented by using the modified ranking scale (mRS).

\section{Implementation of Stroke Unit Care}

The SU manual was developed and was written jointly by the researchers. This manual defines stroke clearly for the staff. It gives guidelines on quick, efficient, and pertinent history taking. It guides the staff on how to rapidly examine a suspected stroke patient, accurately calculate stroke severity, and localize the lesion according to symptoms. A modified multi-MDT was used for post-stroke care including a physician, nurses, physiotherapists, and an occupational therapist. 
It helps in ordering all relevant investigations at baseline. This manual also helped in establishing a geographically defined SU, determining the number of beds according to need, employing MDT working, additional training of the existent staff, and establishing protocols. The MDT met once a week to discuss and plan the management of individual patients. The team also organized seminars for the relatives and caregiversregularly.

Six beds were allocated in SU. Protocols for blood pressure and blood sugar controls were also introduced and implemented. Antiplatelet and anticoagulants guidelines were skills in the area of diagnosis, investigation, and treatment of stroke patients. The team was also trained to address the issues that can affect the patient outcome and emphasized providing MDT approach to improve stroke care. The training module was adapted from the Indian Stroke Association guidelines. ${ }^{8}$ In the second cohort, 125 consecutive stroke patients were reassessed. The same variables in the pre-SU were documented in post-SU care.

The key features of stroke intervention are mentioned in the box below:

Key features of stroke intervention

Training of local physician: 2 hours training session for three times in a week for 1 month through Skype by stroke experts.

- How to establish the SU

- Key components of basic SU care, assessment, and monitoring with selected clinical assessments (neurological and medical monitoring)

- Medical and rehabilitation management in the acute phase

Training of stroke team (SU nurses, physiotherapists, and occupation therapist): locally, the trained physician has conducted 2 hours session for 5 days a week for 1 month to stroke team as guided by stroke rehab experts.

- Acute nursing care

- Secondary complications management

- Importance of the multidisciplinary approach

- Early mobility and post-stroke rehabilitation

- Discharge planning

Formal multidisciplinary team meetings to discuss individual patient progress, treatment goals, and the issues that can affect the patient outcome

Stroke care pathway protocols

Assessment and monitoring

- Medical diagnosis

- Nursing assessment and monitoring

- Treatment assessment
Acute management (first 5 days)

- Physiological management

- Managing complications

- Early mobilization

- Acute nursing care

\section{Multidisciplinary team (MDT)} approach

- Coordinated teamwork

- Close linking of nursing with rehabilitation

- Early rehabilitation and involvement of caregivers

- Provision of information on stroke, recovery, and rehabilitation
Discharge planning

- Early assessment of discharge needs

- Identification of recovery goals with patient and caregiver

- Discharge plan involving patient and caregiver

- Rehabilitation in-home setting

1-month patients' follow-up was taken in outpatient department or telephonically

established. A swallow test was performed on all patients in the SU. If a patient did not pass the swallow test, a nasogastric tube was inserted. All the complications documented in both pre-SU and post-SU groups were defined as per our previously published research. ${ }^{7}$

\section{Post-Stroke Unit Phase}

The SU intervention with protocols was implemented after 1 month of training. A 2-hour training was given to physician for three times in a week for 1 month through Skype. The physician then trained the SU nurses, physiotherapists, and occupational therapist. The training program was designed to promote the core knowledge,
The reporting guidelines of STROBE were followed for the study and the allocation of participants was shown in a flow (-Supplementary Material 1 [available in the online version]).

\section{Sample Size}

The previously done study comparing SU care with patients managed in the medical ward in an Indian setting showed a reduction of complication by $22 \%$ and in-hospital mortality by $35 \% .^{9}$ The sample size for an expected $20 \%$ reduction in complications and a $35 \%$ reduction in-hospital mortality was 210 and 62 , respectively, at $80 \%$ power with a type 1 error of $5 \%$. By taking the maximum of both, the sample size for 
our study was $n=250,125$ in each group considering a $15 \%$ drop out rate.

\section{Statistical Analysis}

In descriptive statistics analysis, continuous variables are stated as mean \pm standard deviation or median (interquartile range). Categorical variables are depicted as count (percentage). Kolmogorov-Smirnov test was used to check the normality of the variables. Chi-square was used to compare the categorical variables and the Student's t-test or MannWhitney $U$ test was used to compare continuous variables between the groups. The loss to follow-up was imputed by using multiple imputations using the Markov Chain Monto Carlo method (MCMC) after missing patterns showed the values were missing at random by treating the functional outcome as a dichotomous variable with 0 to 2 score indicating good outcome and 3 to 5 indicating bad outcome. The sensitivity analysis was also performed by using the propensity score matching of the groups for baseline stroke severity (NIHSS) using the nearest neighbor approach to control for confounding, and missing values were imputed by using multiple imputations. The adjusted odds ratio was calculated in univariate and multivariate regression analysis after adjusting for baseline variables.

All the analysis was done by using SPSS, version 21.0. IBM Corp and R version 4.0.0, Armonk, New York, United States.

\section{Results}

Demography: Total number of stroke patients admitted to the hospital during the study period was 250 , with pre-stroke and post-SU arms having 125 patients each. The mean age of the patients was $61 \pm 14.1$ years and 153 (61\%) were men. There was a difference in education levels and work status between both the groups (-Supplementary Material S2 [available in the online version]).

Patients in pre-SU group had higher NIHSS score. Intracerebral hemorrhage rate was high in both the groups (pre-SU 74 [59.2\%] vs. post-SU care 63 (50.4\%), $p=0.227$. Fewer patients in the pre-SU group had hypertension (77 [61.6\%] vs. 122 [97.6\%], $p \leq 0.001$ ), nonvalvular atrial fibrillation ( $1[0.8 \%]$ vs. 18 [14.4\%]; $p<0.001)$ and diabetes (16 [12.8\%] vs. 19 [23.2\%]; $p=0.032$ ); all other risk factors were comparable (-Supplementary Material S2 [available in the online version]).

In the pre-SU group, the overall incidence of complications was higher as compared with the post-SU group (-Table 1).

A higher proportion of patients in the post-SU group received anticoagulants, antiplatelet, antihypertensive, oral hypoglycemic, and cholesterol-lowering drugs both in-hospital and at discharge as compared with the pre-SU group ( - Table 2 ).

In the post-SU group, the length of hospital stay was shorter, and no difference was observed in functional outcome (mRS) at 1 month after imputation for the lost to follow-up was done by using multiple imputation method. The functional outcome after imputation showed the same
Table 1 Comparison of complications in pre-stroke and poststroke unit groups

\begin{tabular}{|c|c|c|c|}
\hline Complications & $\begin{array}{l}\text { Pre-stroke } \\
\text { unit } \\
n(\%)\end{array}$ & $\begin{array}{l}\text { Post-stroke } \\
\text { unit } \\
n(\%)\end{array}$ & $p$-Value \\
\hline Overall & $56(44.8)$ & $35(28.0)$ & 0.006 \\
\hline \multicolumn{4}{|l|}{ Neurological } \\
\hline Seizure & $1(0.8)$ & $2(1.6)$ & \multirow[t]{2}{*}{0.505} \\
\hline $\begin{array}{l}\text { Unexplained } \\
\text { events }\end{array}$ & $0(0)$ & $1(0.8)$ & \\
\hline \multicolumn{4}{|l|}{ Infection } \\
\hline UTI & $0(0)$ & $2(1.6)$ & \multirow[t]{3}{*}{0.145} \\
\hline Chest infection & $11(8.8)$ & $12(9.6)$ & \\
\hline Other infections & $1(0.8)$ & $6(4.8)$ & \\
\hline \multicolumn{4}{|l|}{ Pain } \\
\hline Shoulder & $2(1.6)$ & $1(0.8)$ & \multirow[t]{2}{*}{0.650} \\
\hline Other & $10(8.0)$ & $9(7.2)$ & \\
\hline \multicolumn{4}{|l|}{ Psychological } \\
\hline Depression & $14(11.2)$ & $0(0)$ & \multirow[t]{3}{*}{0.031} \\
\hline Anxiety & $8(6.4)$ & $1(0.8)$ & \\
\hline Confusion & $18(14.4)$ & $9(7.2)$ & \\
\hline
\end{tabular}

Abbreviation: UTI, urinary tract infection.

trend as in a complete case analysis (adjusted odds ratio [OR]: 0.65; 0.26-1.66, $p$ = 0.369; - Table 3).

The sensitivity analysis was performed by using propensity score matching for baseline severity after missing values imputed using multiple imputation method, but both the groups were found matching for all the observations. After case-mix adjustment for baseline stroke severity, stroke type, first-ever stroke, and risk factors in multivariate logistic regression model, we found more patients in the post-SU group received mobilization (adjusted OR: $4.14 ; 0.33-51.38$ ) and in-hospital secondary stroke preventions, diabetes medication (adjusted OR: 3.18; 0.98-9.90), and statins (adjusted OR: 4.03; 0.40-40.39, $p$ $=0.236$ ) compared with the pre-SU group. The odds ratio for the case-mix adjustment model was shown in a forest plot (-Fig. 2).

\section{Discussion}

The physician-based SU model in a remote area of India showed fewer in-hospital complications, reduced hospital stay, improvement in secondary prevention measures, and a trend toward a better outcome.

The interventions made in $\mathrm{BCH}$, Tezpur did not require large capital investment but appeared to improve the quality of stroke care and patient outcome. It is especially important for LMICs like India where neurologists and imaging facilities are limited specifically in rural areas. The interventions had to incorporate the high proportion of intracerebral hemorrhage and the other components of intervention include supplementing the training of nurses and other staff in the rapid evaluation of stroke severity, localizing lesions and order timely 
Table 2 Comparison of assessment and re-education and secondary stroke prevention given in pre-stroke and post-stroke unit groups

\begin{tabular}{|c|c|c|c|c|c|}
\hline & \multirow{2}{*}{$\begin{array}{l}\text { Pre-stroke } \\
\text { unit } \\
n(\%)\end{array}$} & \multirow{2}{*}{$\begin{array}{l}\text { Post-stroke } \\
\text { unit } \\
n(\%)\end{array}$} & \multirow[t]{2}{*}{$p$-value } & \multicolumn{2}{|c|}{ Adjusted odds ratio } \\
\hline & & & & $\begin{array}{l}\text { Odds } \\
\text { ratio }\end{array}$ & 95\% Cl (lower-upper) \\
\hline \multicolumn{6}{|c|}{ Assessment and re-education } \\
\hline Swallowing assessment & $0(0)$ & $46(36.8)$ & $<0.001$ & & \\
\hline $\begin{array}{l}\text { Mobility assessment and } \\
\text { re-education }\end{array}$ & $114(91.2)$ & $124(99.2)$ & 0.037 & 10.034 & $1.152-87.399$ \\
\hline $\begin{array}{l}\text { Evidence of meeting with } \\
\text { multidisciplinary team } \\
\text { approach }\end{array}$ & $114(91.2)$ & $125(100.0)$ & 0.001 & & \\
\hline \multicolumn{6}{|c|}{ In hospital (secondary stroke prevention) } \\
\hline Anticoagulant drugs & $1(0.8)$ & $17(13.6)$ & $<0.001$ & 10.346 & $1.299-82.413$ \\
\hline Antiplatelet drugs & $0(0)$ & $39(31.2)$ & $<0.001$ & & \\
\hline Antihypertensive & $103(83.1)$ & $121(96.8)$ & $<0.001$ & 4.773 & $1.392-16.364$ \\
\hline Diabetes medication & $8(6.4)$ & $27(21.6)$ & 0.001 & 8.336 & $1.826-37.842$ \\
\hline Statins & $1(0.8)$ & $9(7.2)$ & 0.010 & 4.094 & $0.476-35.192$ \\
\hline \multicolumn{6}{|l|}{ On discharge } \\
\hline Anticoagulant drugs & $0(0)$ & $18(14.4)$ & $<0.001$ & & \\
\hline Antiplatelet drugs & $1(0.8)$ & $33(26.4)$ & $<0.001$ & 42.447 & $4.669-383.427$ \\
\hline Antihypertensive & $82(65.6)$ & $113(90.4)$ & $<0.001$ & 4.632 & $2.124-10.099$ \\
\hline Diabetes medication & $7(5.6)$ & $30(24.0)$ & $<0.001$ & 21.108 & $4.279-104.121$ \\
\hline Statins & $0(0)$ & $10(8.0)$ & 0.001 & & \\
\hline
\end{tabular}

Table 3 Comparison of outcome at discharge and after 1 month in pre-stroke unit and post-stroke unit groups

\begin{tabular}{|c|c|c|c|c|c|}
\hline \multirow[t]{2}{*}{ Outcome } & \multirow{2}{*}{$\begin{array}{l}\text { Pre-stroke unit } \\
n(\%)\end{array}$} & \multirow{2}{*}{$\begin{array}{l}\text { Post-stroke unit } \\
n(\%)\end{array}$} & \multirow[t]{2}{*}{$p$-Value } & \multicolumn{2}{|c|}{ Adjusted odds ratio } \\
\hline & & & & Odds ratio & 95\% Cl (lower-upper) \\
\hline $\begin{array}{l}\text { Length of hospital stay }(\mathrm{d}) \\
\text { Mean } \pm \text { SD }\end{array}$ & $5 \pm 2.68$ & $4 \pm 2.16$ & 0.026 & 0.039 & $0.011-0.133$ \\
\hline \multicolumn{6}{|l|}{ Vital status at discharge } \\
\hline Alive & $114(91.2)$ & $121(96.8)$ & 0.062 & 1.000 & \\
\hline Dead & $11(8.8)$ & $4(3.2)$ & & 0.376 & $0.091-1.543$ \\
\hline Mortality & $13(10.4)$ & $19(15.2)$ & 0.256 & 2.391 & $0.862-6.627$ \\
\hline \multicolumn{6}{|l|}{$\mathrm{mRS}$} \\
\hline Good outcome (0-2) & $40(35.7)$ & $42(39.6)$ & 0.552 & 0.732 & $0.274-1.956$ \\
\hline Bad outcome (3-5) & $72(64.3)$ & $64(60.4)$ & & 1.000 & \\
\hline
\end{tabular}

Abbreviations: $\mathrm{Cl}$, confidence interval; mRS, modified ranking scale; SD, standard deviation.

investigations, maintain blood pressure and blood sugar levels, holding a weekly assessment meeting to plan individual patient care, and train the caregiver and/or relative while the patient was admitted in the hospital.

In a study from South Africa, multidisciplinary stroke care was successfully implemented in a secondary-level hospital with a reduction in inpatient mortality and an increase in referral for inpatient rehabilitation..$^{10}$ In a few states of India, physician-based SUs have been developed with subsequent increases in the use of thrombolysis; however, there was no comparison made before and after implementation of SUs. ${ }^{11}$ Besides, this model of care emphasized thrombolysis more than SU care. Organized stroke care across income levels
(OSCAIL) is a similar initiative, which is being tested in multiple remote hospitals in Africa and India where there is a lack of infrastructure. The northeastern states in India have a high rate of stroke in particular $\mathrm{ICH}^{2,12,13}$ This is related to the high prevalence of hypertension in these regions. ${ }^{2}$ In the post-SU group, the higher use of ECG could have been the reason which leads to greater cases of atrial fibrillation.

This study has several strengths; it was done in a remote rural setting where there are no neurologists. The physician-based model is feasible and can lead to an improvement in stroke quality outcome measures. Reorganizing the existing infrastructure of the hospital helped to avoid the huge costs involved in setting up SUs. Lack of infrastructure 


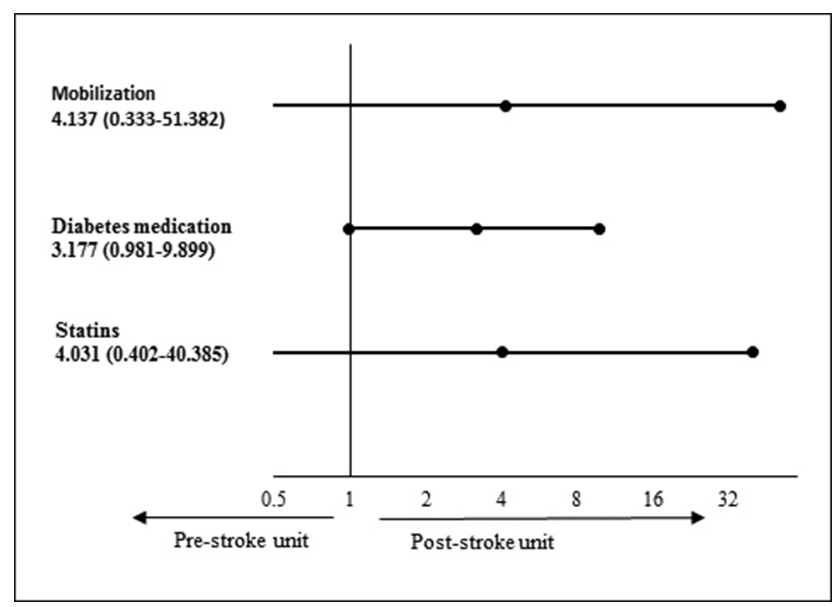

Fig. 2 Shows a forest plot for multivariate logistic regression after adjusted for baseline stroke severity, stroke type, first-ever stroke, and risk factors.

is a major challenge in establishing SUs in LMICs which this study was able to address and successfully implement.

The study limitation is that it was a before and after design study which tests the short-term effects of an intervention. In remote areas in India, it is difficult sometimes to follow up the patients due to multiple factors. Hence, we imputed the missing values using a multiple imputation method. Missing value analysis of the data showed a random pattern, which indicates that the outcome of this study will not be significantly influenced by missing values. Despite the above limitation, this study appeared to show improved quality outcome measures in SU care.

\section{Conclusion}

A physician-based SU model is feasible in a remote area in India and showed improved quality measures. The implementation of this model should be feasible for other remote hospitals within the country and also in other LMICs.

\section{Note}

All data relevant to the study are included in the article or uploaded as supplementary information. The raw data used study has been uploaded and cited in the reference. ${ }^{14}$ Abstract submission for poster presentation at World Stroke Congress, 2018, Montreal, Canada.

The approval for the study was obtained from institutional review board of Emmanuel Hospital Association. The informed written consents were taken from all the participants of the study.

\section{Funding}

This work was supported by Institute of Cardiovascular and Medical Sciences, Royal Infirmary Hospital, Glasgow.

\section{Authors' Contributions}

J.D.P. and P.L. designed the study and manual and reviewed the manuscript. J.D.P. was involved in the training and implementation of the study. L.J. and A.G.W. wrote and reviewed the manuscript, and H.K. assisted statistical design. All authors reviewed and edited the manuscript and approved the final version of the manuscript.

\section{References}

1 Johnson CO, Nguyen M, Roth GA, et al; GBD 2016 Stroke Collaborators. Global, regional, and national burden of stroke, 1990-2016: a systematic analysis for the Global Burden of Disease Study 2016. Lancet Neurol 2019;18(5):439-458

2 Prabhakaran D, Jeemon P, Sharma M, et al. The changing patterns of cardiovascular diseases and their risk factors in the states of India: the Global Burden of Disease Study 1990-2016. The Lancet Global Health. 2018;6(12):e1339-e1351

3 Langhorne P, de Villiers L, Pandian JD. Applicability of stroke-unit care to low-income and middle-income countries. Lancet Neurol 2012;11(4):341-348

4 Baatiema L, de-Graft Aikins A, Sav A, Mnatzaganian G, Chan CKY, Somerset S. Barriers to evidence-based acute stroke care in Ghana: a qualitative study on the perspectives of stroke care professionals. BMJ Open 2017;7(4):e015385

5 Pandian JD, William AG, Kate MP, et al. Strategies to improve stroke care services in low- and middle-income countries: a systematic review. neuroepidemiology 2017;49(1-2):4561 pubmed

6 Suwanwela NC, Poungvarin N; Asian Stroke Advisory Panel. Stroke burden and stroke care system in Asia. Neurol India 2016;64(7, Suppl):S46-S51

7 Pandian JD, Kaur A, Jyotsna R, et al. Complications in acute stroke in India (CAST-I): a multicenter study. J Stroke Cerebrovasc Dis 2012;21(8):695-703

8 Khurana D, Padma MV, Bhatia R, et al. Recommendations for the early management of acute ischemic stroke: a consensus statement for healthcare professionals from the Indian Stroke Association. J Stroke Med 2018;1(2):79-113

9 Pandian JD, Joy SA, Justin M, et al. Impact of stroke unit care: an Indian perspective. Int J Stroke 2011;6(4):372-373

10 de Villiers L, Kalula SZ, Burch VC. Does multidisciplinary stroke care improve outcome in a secondary-level hospital in South Africa? Int J Stroke 2009;4(2):89-93

11 Sharma S, Padma MV, Bhardwaj A, Sharma A, Sawal N, Thakur S. Telestroke in resource-poor developing country model. Neurol India 2016;64(5):934-940

12 India State-Level Disease Burden Initiative Collaborators. Nations within a nation: variations in epidemiological transition across the states of India, 1990-2016 in the Global Burden of Disease Study. Lancet 2017;390(10111):2437-2460

13 Das SK, Banerjee TK, Biswas A, et al. A prospective communitybased study of stroke in Kolkata, India. Stroke 2007;38(3): 906-910

14 Pandian J, Implementation of a physician-based stroke unit in a remote hospital of North-East India-Tezpur model, v2, Dryad, Dataset 2020;10.5061/dryad.2bvq83bm7*Give information separately for cases and controls in case-control studies and, if applicable, for exposed and unexposed groups in cohort and cross-sectional studies

\section{Conflict of Interest}

None declared. 